\title{
Properties of Visible Lattice Points
}

\section{Teodoro B. Mendes*, Artem Lopatin}

\begin{abstract}
This project aims to expose some properties about lattice points. More specifically, these properties are patterns on the visible lattice points (visible from the perspective of a point located at the origin of the lattice) that can be modeled by the use of the Chinese Remainder Theorem. These patterns also opens a wide spectrum of questions about the nature of prime numbers and how intimately related they are.
\end{abstract}

\section{Key words:}

\section{Lattice, CRT, coprime}

A lattice is an algebraic structure which consists of a subgroup for any basis of $\mathbf{R}^{\mathbf{n}}$ of all linear combinations with integer coefficients between these basis. On this project, we work on the $\mathbf{R}^{2}$ lattice with its standard basis.

The visible points of this lattice are simply the points $(\mathbf{a}, \mathbf{b})$ where $\mathbf{a}$ and $\mathbf{b}$ are coprime, in other words, $\operatorname{gdc}(\mathbf{a}, \mathbf{b})=\mathbf{1}$. These points are called visible (from the origin) simply because the line that connects the origin and the mentioned points $(\mathbf{a}, \mathbf{b})$, contains no other points, since they are coprime ${ }^{1}$.

This project defines and presents some properties about "visible squares" contained in the lattice. A visible square (only its border is considered), also called "black square" is a set $\mathbf{Q}(\mathbf{i}, \mathbf{j}, \mathbf{c})$ where $(\mathbf{i}, \mathbf{j})$ is the center of the square and c is the lower distance from the center to the square border, which means that the size of the sides of the square is $\mathbf{2 c + 1}$.

In particular, we present properties about the sets $\mathbf{Q}(\mathbf{i}, \mathbf{j}, \mathbf{1})$ and $\mathbf{Q}(\mathbf{i}, \mathbf{j}, \mathbf{2})$ that implies the infinitude of them.

\section{Results and Discussion}

A trivial lemma that needs to be satisfied for every $\mathbf{Q}$ set is that the sides of the black squares (elements of $\mathbf{Q}$ ) are composed by odd numbers.

For the $\mathbf{Q}(\mathbf{i}, \mathbf{j}, \mathbf{1})$ set we could find two lemmas that together come up with the expected solution. The first one states that a $\mathbf{3} \times \mathbf{3}$ square $\mathbf{Q}(\mathbf{r}, \mathbf{s}, \mathbf{1})$ is black if, and only if, $\mathbf{6 | r}$. Also, for a decomposition of $\mathbf{r - 1}, \mathbf{r}$ and $\mathbf{r + 1}$ in prime numbers, there is a solution to $\mathbf{s}$ that implies the existence of at least one black square of size $3 \times 3$. The second lemma states that there is an infinite number of solutions to $\mathbf{s}$ and, therefore, that the set $\mathbf{Q}(\mathbf{i}, \mathbf{j}, \mathbf{1})$ is infinite.

The path for solving the same problem to the $\mathbf{Q}(\mathbf{i}, \mathbf{j}, \mathbf{2})$ set is pretty much analogous to the one explained above, so we omit it.
The images below contain a visual representation of what has been presented and explained here.

The origin is the most left point of the bottom line. Note that these pictures could be extended for all four quadrants, this is only the first quadrant representation.

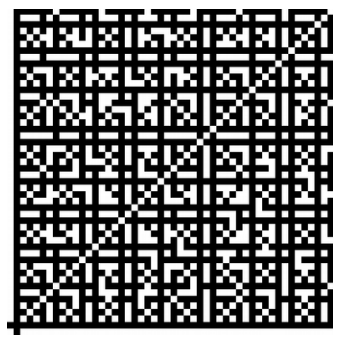

Image 1. Picture of visible lattice points (50x50 points).

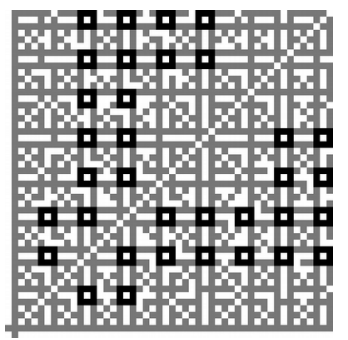

Image 2. Highlighted black squares of $\mathbf{Q}(\mathbf{i}, \mathbf{j}, \mathbf{1})$.

\section{Conclusions}

We could prove, by the use of the Chinese Remainder Theorem that the sets $\mathbf{Q}(\mathbf{i}, \mathbf{j}, \mathbf{1})$ and $\mathbf{Q}(\mathbf{i}, \mathbf{j}, \mathbf{2})$ contains an infinite amount of elements. We found a system of congruences, for each one of these sets, that has infinite solutions. Therefore, the sets are infinite. However, nothing could be talked about a general formula for finding the elements of these sets based on the value of $\mathbf{c}$ (i.e. the black squares of any size) because this would imply a formula for finding prime numbers, which would probably require a very longer time of research to find it.

\section{Acknowledgement}

We thank PIBIC and UNICAMP for giving us this research program opportunity.

\footnotetext{
${ }^{1} \mathrm{~A}$. Mbirika, Hidden trees in the forest: On lattice points and prime labelings of graphs, Bowdoin College (2012), 3-16.
} 